\title{
Interactions between microfibrillar cellulose and carboxymethyl cellulose in an aqueous suspension
}

Deepa Agarwal, William MacNaughtan, and Tim J. Foster*

Division of Food Sciences, School of Biosciences, University of Nottingham, Sutton

5 Bonington Campus, Loughborough, LE12 5RD, UK.

6

7

\section{Abstract}

9 New microstructures with interesting, unique and stable textures, particularly relevant to food systems were created by redispersing Microfibrillar cellulose (MFC). This paper reports the interactions between microfibrillar cellulose and carboxymethyl cellulose (CMC) in redispersed aqueous suspensions, by using rheological measurements on variable ratios of MFC/CMC and correlating these with apparent water mobility as determined by time domain NMR. MFC is a network of cellulose fibrils produced by subjecting pure cellulose pulp to

*Corresponding author e-mail: tim.foster@nottingham.ac.uk high-pressure mechanical homogenisation. A charged polymer such as CMC reduces the aggregation of microfibrillar/fibre bundles upon drying. Small amplitude oscillatory rheological analysis showed the viscoelastic gel-like behaviour of suspensions which was independent of the CMC content in the MFC suspension. A viscous synergistic effect was observed when CMC was added to MFC before drying, leading to improved redispersibility of the suspension. Novel measurements of NMR relaxation suggested that the aggregated microfibrillar/fibre bundles normally dominate the relaxation times $\left(T_{2}\right)$. The dense microfibrillar network plays an important role in generating stable rheological properties and controlling the mobility of the polymer and hence the apparent mobility of the water in the suspensions.

\section{Highlights}


- $\mathrm{CMC}$ improves redispersibility and reduces aggregation of MFC microfibrils

- NMR relaxation measurements give an insight into the mechanisms of redispersibility

- Polymer aggregation dominates the $\mathrm{T}_{2}$ value and NMR behaviour of suspensions

- Improved re-dispersion is correlated with higher shear viscosity and increased $\mathrm{T}_{2}$

- Unique microstructures relevant to foods have been created

Keywords: Microfibrillar cellulose; carboxymethyl cellulose; low-field NMR; relaxation time; rheology

\section{Introduction}

Cellulose is the most abundant natural structural polymer in nature and provides mechanical properties such as strength and stiffness to the plant cell wall of higher plants. Important components of this natural fibre strength and stiffness are the microfibrils within the cellulose structure. The fibrous cell wall is essentially a composite material consisting of a framework of cellulose (micro-) fibrils organised into strands of cellulose which are embedded in a matrix of hemicelluloses and lignin. Cellulose microfibrils in the cell wall are intertwined fibrils with a diameter of approx. 2-20nm and a length of 100-40,000 nm depending on the source (Kirk and Othmer, 1967; Kocherbitov, Ulvenland, Kober and Jarring, 2008). These cellulose fibres can be broken down into their structural micro/nano-scale units by various chemical and mechanical processes (Henriksson, Berglund and Lindstrom, 2007). Production and characterisation of microfibrillar cellulose (MFC) from wood fibres have been described by Turbak et al. 1983 and Herrick et al. 1983, where MFC suspensions were obtained by disintegrating cellulose fibres at high shear. The resultant highly entangled MFC network consists of micro/nano size elements with a gel-like behaviour for water suspensions at $1 \%$ or lower concentrations of MFC (Turbak et al., 1983, Herrick et al., 1983, Nakagaito and Yano 2004, Nishiyama, 2009). During the last decade, microfibrillar cellulose (MFC) has been produced by using more aggressive, high shear or high energy mechanical treatments such as 
homogenisers or microfluidisers which led to highly entangled, fibril aggregates and mechanically strong networks (Frone et al., 2011, Lavoine et al., 2012). Depending on the pressure, flow rate, temperature, and the design and diameter of the chambers used in highpressure homogenisers or microfluidisers, different particle size distributions and microfibrillar networks can be produced (Lavoine et al., 2012). Several publications have shown applications of these highly networked MFC microfibrils for various purposes, such as reinforcement in nanocomposites (Malainine, Mahrouz and Dufresne 2005, Lopez-Rubio et al., 2007, Bruce et al., 2005), dispersion stabilization (Oza and Frank 1986, Ougiya et al., 1997, Khopade and Jain 1990), media filtration (Burger, Hsiao and Chu 2006), antimicrobial action in films (Andresen et al., 2007) and oxygen barrier production in food and pharmaceuticals (Syverud and Stenius 2009). The rheological properties of these MFC suspensions have been widely studied by a number of researchers. In general, the rheological properties of aqueous MFC suspensions isolated from softwood, sugar beet pulp, corn cobs and cotton show gel-like behaviour where the storage modulus $\left(G^{\prime}\right)$ is higher than the loss modulus (G”) over a wide concentration range (Pääkkö et al., 2007, Tanjawa et al., 2010, Cordabo et al., 2010, Tatsumi et al., 2002, Tatsumi et al., 2007).

Homogenisation modifies the structure of the starting materials by releasing microfibrils into the suspension. Drying the MFC is also known to modify the defibrillated state primarily by increased hydrogen bonding but possibly also other forms of bonding such as van der Waals between the microfibrils, leading to the formation of bundles and agglomerates (Quiévy et al., 2010). These fibre bundles and aggregates are difficult to redisperse in water in order to form homogeneous suspensions, a consequence being a reduction in the values of rheological parameters such as G', G' and the shear viscosity of the suspension. This process of irreversible or partial irreversible agglomeration of cellulosic fibres and stiffening of the polymer structure during drying is known in the literature as hornification. It is a technical term widely used in the paper-making industry (Smook 1990, Kato et al., 1999, Fernandes et al., 2004). The 
aggregation or agglomeration occurs to varying extents depending on the drying process. To protect the microfibrils from collapse and agglomeration, a number of hydrocolloids, e.g. low and high methoxyl pectin, CMC, and sodium polyacrylate, as well as salts $e$.g. sodium chloride (Lowys, Desbrieres \& Rinaudo, 2001; Tandjawa et al., 2012; Missoum, Bras \& Belgacem, 2012), have been used to stabilise the fibrils. Lowys (2001) demonstrated an interaction between MFC and polymeric additives such as sodium-CMC and pectins, where the additives were homogeneously distributed and formed weak bonds with MFC fibres improving the redispersibility of MFC in water. This interaction between the additive and MFC tends to stabilise the fibrils against collapse or agglomeration during the drying process. The objective of the current publication is to provide an insight into the impact of drying (hornification) on the state of the polymer and the apparent water mobility in the MFC matrix.

Rheological properties of aqueous suspensions of MFC with or without additives show viscoelastic gel-like behaviour and high viscosity (Cordabo et al., 2010, Agoda-Tandjawa et al., 2010). Such properties of aqueous suspensions at $1 \%(\mathrm{w} / \mathrm{w})$ and lower concentrations, make MFC valuable in a wide range of industrial applications such as food, cosmetics, paints and composites, etc. The strong interactions between the MFC fibres in aqueous media are the driving force behind rheological characteristics, such as water binding and viscosity. AgodaTandjawa (2012) reported that in the presence of calcium ions, low methoxyl pectin exhibited a synergistic effect with MFC fibres leading to increased shear and complex viscosities of the composites. In the present study, the impact of carboxymethyl cellulose on rheological properties of a dried and redispersed MFC suspension was studied.

It has been suggested that proton nuclear magnetic resonance (NMR) parameters such as spinlattice-relaxation time $\left(T_{1}\right)$ and spin-spin relaxation time $\left(T_{2}\right)$ are sensitive to water state and mobility in polymeric suspensions/dispersions (Ono, Inamoto and Okajima, 1997; Rachocki, Markiewicz and Tritt, 2005, Vackier, Hills and Rutledge, 1999). The spin-spin relaxation time 
$\mathrm{T}_{2}$ is generally measured using the Carr-Purcell-Meiboom-Gill (CPMG) pulse sequence

103 (Meiboom and Gill, 1958). The CPMG sequence provides a more accurate measure of the

104

105

106

107

108

109

110

111

112

113

114

115 liquid transverse free-induction decay time $\left(\mathrm{T}_{2}\right)$ and is free of artefacts such as magnetic inhomogeneity. In a study by Ono (1997), an MCC suspension was shown to contain both freewater and water-associated to the polymer with a mutual exchange of protons resulting in shorter overall $\mathrm{T}_{2}$ compared to pure distilled water, where a typical $\mathrm{T}_{2}$ is of the order of 2 seconds.

The primary aim of this study then is focused on understanding the impact of CMC on the redispersibility of MFC in water and its impact on rheological properties of the suspension. It is hoped that this understanding will shed light on the occurrence of aggregation of MFC and the technical problems that ensue from this in various industries from food to paper-making. A detailed study of rheological behaviour and the NMR determined apparent water mobility of the redispersed MFC/CMC system, when correlated with fluorescence microscopy, as presented here, will enable important structural features of these cellulosic materials which are of relevance to the food and personal care industries to be determined. The hypothesis underpinning this research is that the addition of CMC to an MFC suspension improves the redispersibility of MFC after drying, by increasing the repulsion between polymer chains due to the charge on the added polymer, and that the effects on the apparent water mobility in the matrix are ultimately due to this.

\section{Materials and methods}

\subsection{Materials}

Microfibrillar cellulose (MFC) from spruce cellulose (8.97\%w/w MFC paste) was provided by Borregaard AS (Sarpsborg, Norway). Cellulose was obtained from 100\% spruce. The charge density of pure cellulose changes noticeably during the pre-treatment and finishing process to produce MFC (Ribitsch et al., 2001). From the information provided by the supplier, the charge 
127 density on the microfibrillar cellulose will be low. Carboxymethyl cellulose (CMC) with a

128 degree of substitution of 0.71 was supplied by CP Kelco (Norway). Reverse osmosis (RO) 129 water was used for all experiments. Light mineral oil density $0.838 \mathrm{~g} / \mathrm{mL}$ at $25^{\circ} \mathrm{C}$ (Sigma130 Aldrich, UK) was used during the rheological measurements to prevent sample dehydration.

\subsection{Sample preparation and biopolymer mixtures}

$2 \% \mathrm{w} / \mathrm{w}$ aqueous suspensions of microfibrillar cellulose were prepared by diluting the MFC stock solution $(8.97 \%$ w/w MFC paste) with RO water using a high shear overhead mixer (Silverson, UK) at $8000 \mathrm{rpm}$ for 5minutes. An aqueous solution of CMC $(2 \% \mathrm{w} / \mathrm{w})$ was prepared separately and added to a $2 \% \mathrm{w} / \mathrm{w}$ MFC suspension according to the formulations shown in Table 1, to produce an overall concentration including both components of $2 \%$. The CMC sample was dissolved by dispersing in $\mathrm{RO}$ water $(2 \% \mathrm{w} / \mathrm{w})$ under gentle stirring (IKA Eurostar 20 Digital Overhead Stirrer) at room temperature for $2 \mathrm{~h}$. The $\mathrm{pH}$ of the solution was adjusted to 6.8 and left overnight at $4^{\circ} \mathrm{C}$ before mixing with the MFC stock suspension. Sodium azide solution $(0.02 \% \mathrm{w} / \mathrm{w})$ was added to prevent bacterial contamination. The concentration of stock samples was determined by evaporating to dryness and measuring the dry solids content.

Table 1: Composition of the MFC/CMC model systems used in this study.

\begin{tabular}{ccc}
\hline \multirow{2}{*}{ Sample Code } & \multicolumn{2}{c}{ \% w/w in suspension } \\
\cline { 2 - 3 } & MFC (\%) & CMC (\%) \\
MFC100 & 2 & 0 \\
CMC15 & 1.7 & 0.3 \\
CMC25 & 1.5 & 0.5 \\
CMC50 & 1 & 1 \\
\hline MFC/CMC solutions were mixed in different proportions as shown in Table 1 at room
\end{tabular}

$144 \mathrm{MFC/CMC}$ solutions were mixed in different proportions as shown in Table 1 at room

145 temperature in water and at an overall concentration of $2 \% \mathrm{w} / \mathrm{w}$. All samples were mixed thoroughly using an overhead stirrer (Silverson, UK) at 8000rpm for 5minutes. The mixtures 
re-dispersion studies, an approximately $1 \mathrm{~mm}$ thin layer of the suspension was layered on an

149 aluminium plate and dried at $50^{\circ} \mathrm{C}$ for 12 hours using a conventional oven (Gallenkamp hotbox oven, size 2).

151 For rheological and relaxation NMR measurements all dry samples were redispersed at 2\%w/w concentration in water by using high shear (T25 digital Ultra-Turrax ${ }^{\circledR}$ ) at 15000rpm for 4minutes at room temperature. Samples were stored overnight at room temperature on a roller bed (Stuart Digital tube rollers - SRT6D) at a speed 60rpm in order to achieve a homogeneous suspension. For relaxation NMR v/s shear viscosity curves, MFC100 and CMC15 "never-dry" (ND) and "dried" (D) suspensions at $0.2-2 \%$ w/w were prepared in RO-water using high shear (T25 digital Ultra-Turrax ${ }^{\circledR}$ ) at $15000 \mathrm{rpm}$ for 4 minutes at room temperature. The $\mathrm{pH}$ of all suspensions was maintained at 6.8 .

\subsection{Rheological measurements}

The rheological measurements were carried out on a stress-controlled rheometer (Physica MCR 301, Anton Paar, Austria) with a serrated parallel plate geometry (50mm diameter with a gap of $1 \mathrm{~mm}$ ) at $20 \pm 1^{\circ} \mathrm{C}$, controlled by a Peltier system. Small oscillatory amplitude sweeps were generated by $\log$ ramping strain 0.01 to $100 \%$ at a constant frequency of $1 \mathrm{~Hz}$. Frequency sweeps were performed over the frequency range of $0.1-15 \mathrm{~Hz}$ at a constant strain of $0.2 \%$ which lay within the linear viscoelastic region. Shear viscosity was measured at constant shear rate i.e. at $50 \mathrm{~s}^{-1}$ at $20 \pm 1^{\circ} \mathrm{C}$. Temperature sweeps were generated by heating the sample between the plates from $20^{\circ} \mathrm{C}$ to $90^{\circ} \mathrm{C}$ at the rate of $1^{\circ} \mathrm{C} / \mathrm{min}$. During these experiments, the strain was fixed at $1 \%$ and the frequency at $1 \mathrm{~Hz}$. A light mineral oil barrier was used to prevent water evaporation. Data presented are an average of four replicates. 
171 Time domain measurements were carried out at $25 \mathrm{MHz}$ using a Resonance Instruments (RI)

172 Maran benchtop NMR spectrometer (Oxford-Instruments Plc, UK). This type of instrument is

173

174

175

176

177

178

179

180

181

182 used routinely in the food industry for fat and moisture measurements. The temperature was regulated at $20 \pm 1^{\circ} \mathrm{C}$ by a conventional gas flow system calibrated with an external thermocouple and controlled with a standard R.I. temperature unit. All measurements were made in $10 \mathrm{~mm}$ outer diameter (OD) NMR tubes. Spin-spin relaxation times $\left(\mathrm{T}_{2}\right)$ were recorded using the CPMG (Curr-Purcell-Meiboom-Gill) pulse sequence (Meiboom and Gill, 1958), $90^{\circ} \mathrm{x}^{---}\left(--\tau--180^{\circ}{ }_{\mathrm{y}}-\tau-\text { echo-- }\right)_{\mathrm{ns}}$ with $\tau=2048 \mu \mathrm{s}$. Typical $90^{\circ}$ pulse lengths were of the order of $5 \mu$ s and $180^{\circ}$ pulse length was $10 \mu$ s. The recycle delay time was fixed at 10 seconds ensuring that all samples were relaxed before the next pulse sequence was applied. 64 scans were recorded. All samples were left at a constant temperature for $15 \mathrm{~min}$ to ensure the temperature was equilibrated and consistent for all data points (see McConville, Pope, 2001). All relaxation curves obtained by the CPMG method showed a single exponential decay.

\subsection{Microscopic analysis}

Light microscopy of aqueous suspensions of samples was performed using an Olympus BX5 bright field light microscope at 20X magnification with a scale bar of $200 \mu \mathrm{m}$. The fibres were dyed using Congo red dye (Sigma-Aldrich). Fluorescence microscopy was carried out using an EVOS microscopy system in fluorescence mode with a 20X objective. As both MFC and CMC do not fluoresce, it was necessary to attach a fluorescence label to one of them. In the current study, CMC was tagged with FITC fluorescent dye. 1g of CMC was dissolved in 10ml of dimethyl sulphoxide containing a few drops of pyridine. $0.1 \mathrm{~g}$ of Isothiocyanate -fluorescein was added to $20 \mathrm{mg}$ dibutyltin dilaurate and the whole mixture was heated at $95^{\circ} \mathrm{C}$ for 2 hours. Free dye was removed from the system by a number of precipitations in ethanol, then the FITC$\mathrm{CMC}$ was filtered and dried at $80^{\circ} \mathrm{C}$. The protocol used is the same as that published by Belder et al., 1973.

\section{Results and Discussion}




\subsection{Viscoelastic properties of MFC/CMC suspensions}

198 Figure $1 \mathrm{~A}$ shows the viscoelastic properties as a function of frequency at $20^{\circ} \mathrm{C}$ for rehydrated aqueous suspensions of MFC/CMC with various contents of CMC (CMC15, CMC25 and CMC50), at a total biopolymer concentration of $2 \% \mathrm{w} / \mathrm{w}$. The storage modulus ( $\left.\mathrm{G}^{\prime}\right)$ of the suspension was higher than the loss modulus (G”) with little dependency on frequency indicating viscoelastic gel-like behaviour. Both moduli increased with increasing frequency, indicating that the network structure formed by the microfibrils is in the dynamic mode of forming entanglements resulting in a stable network of fibres. Similar viscoelastic gel-like behaviour was also observed with never-dried MFC100 and MFC/CMC suspensions. Frequency sweep data for these systems are not shown. Similar viscoelastic behaviour was observed with aqueous suspensions of softwood MFC containing polymeric additives such as pectin, cationic starch etc. (Lowys, Desbrieres and Rinaudo, 2001; Tandjawa et al., 2012). Redispersed MFC/CMC suspensions showed noticeably higher values for G' and G" compared with MFC100 (Figure 1A). Visually, it was observed that the addition of CMC improved the redispersibility of the MFC in water and a homogenous suspension was produced using a high shear mixing process. Figure 1B shows the change in complex viscosity measured at $0.2 \%$ strain and $1 \mathrm{~Hz}$ frequency for pure MFC100 (D) without additives and MFC/CMC mixtures, as a function of CMC proportion in the mixture. In Figure 1B, the concentration for pure MFC100 is identical to that present in MFC/CMC mixtures. It was observed that the complex viscosity $\left(\left|\Pi^{*}\right|\right)$ of the redispersed suspension increased with an increase in CMC proportion in the formulation, indicating that the MFC forms entangled networks crosslinked with CMC, resulting in higher complex viscosity (Figure 1B) and higher values for G' and G' (Figure 1A). 
(A) 1000

224

225

226

227

228

229

230

231

232

233

234

235

236
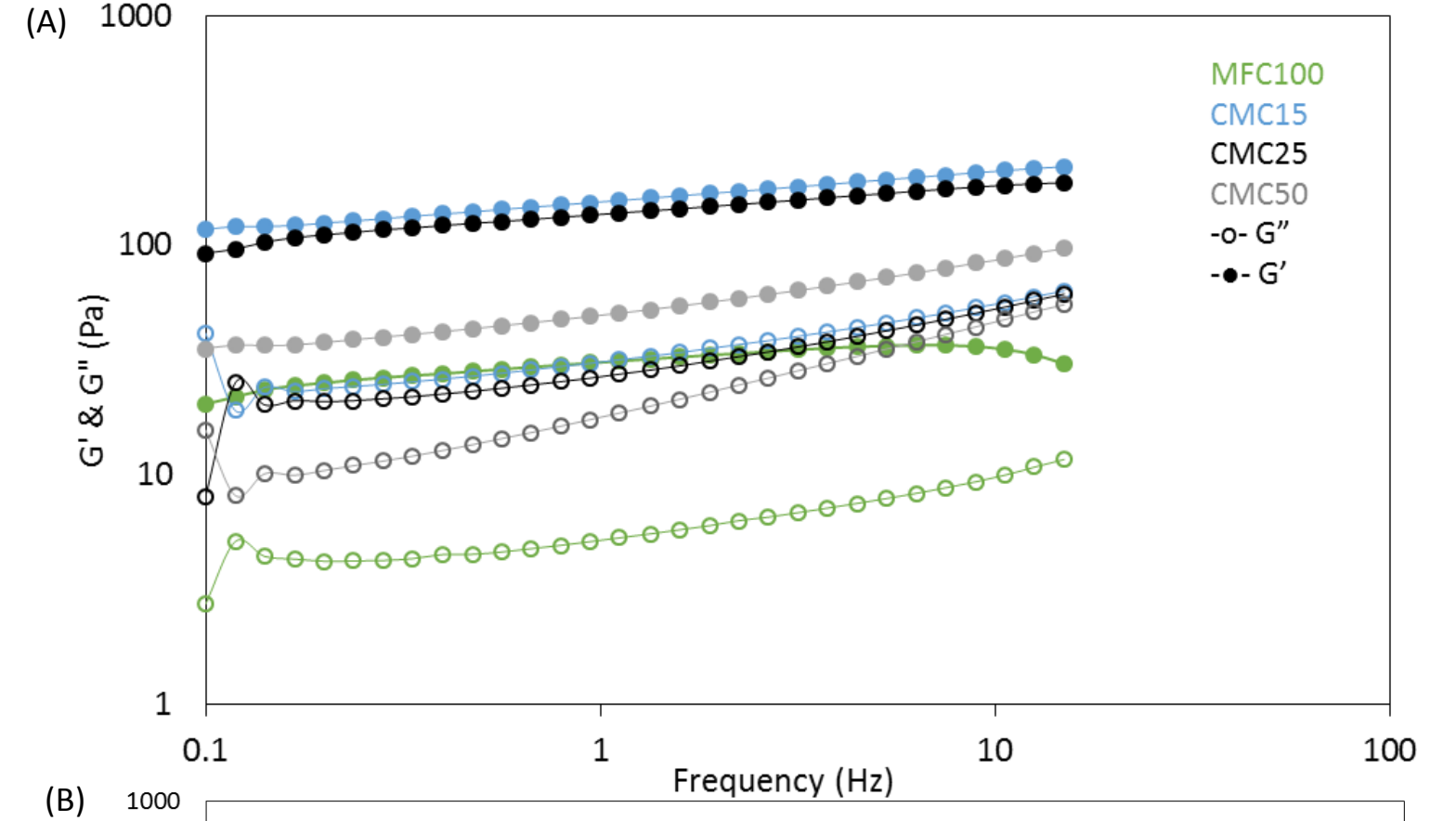

(B) $1 0 0 0 \longdiv { 1 } \quad$ Frequency $(\mathrm{Hz})$

4

35

36

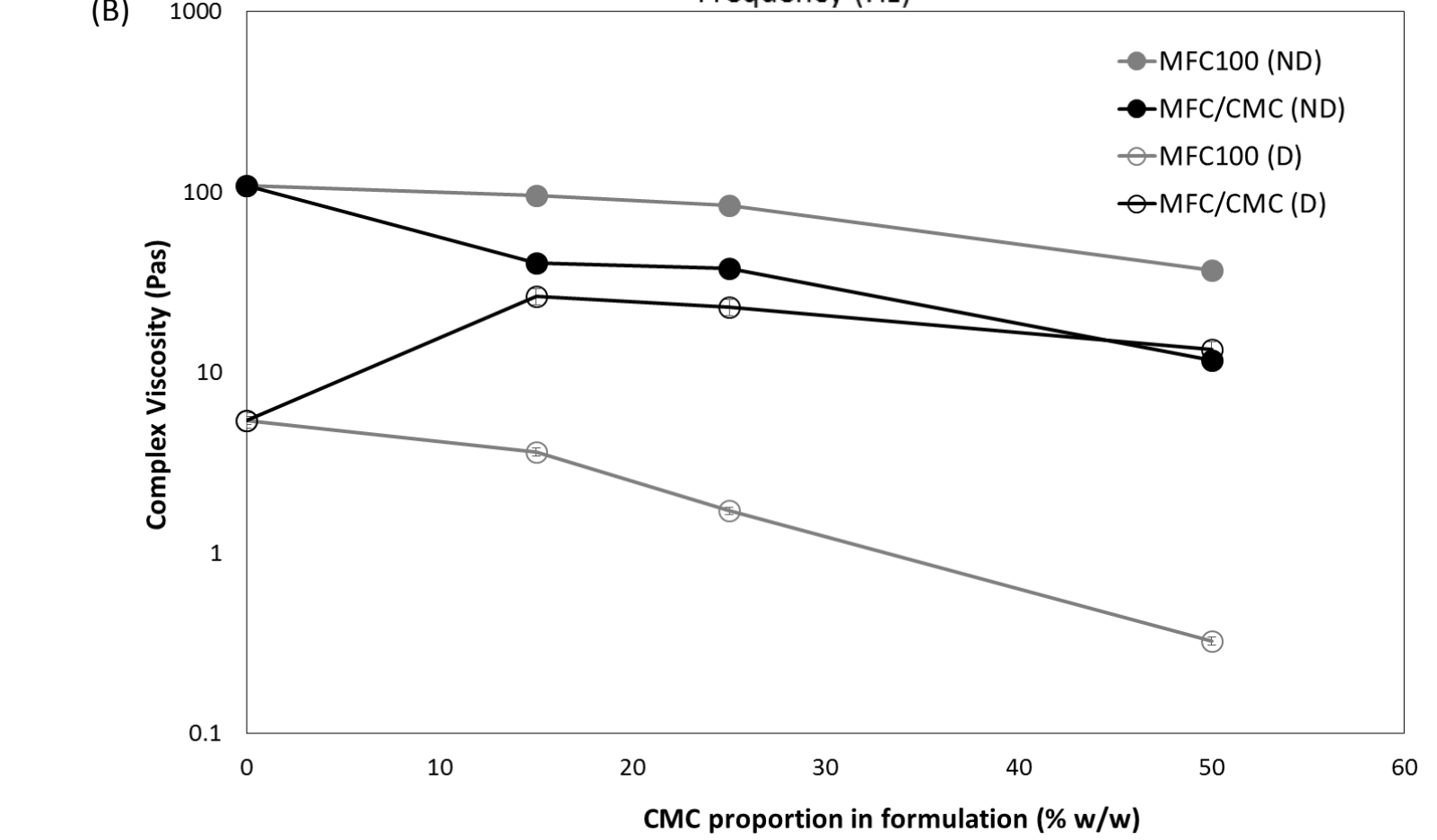

243 Figure 1 (A) Frequency dependency of viscoelastic moduli for MFC/CMC mixtures dried and

244 redispersed in aqueous media. Data were acquired at $0.2 \%$ strain and $20^{\circ} \mathrm{C}$. Solid symbols

245 represent the storage modulus $\left(G^{\prime}\right)$ and open symbols the loss modulus ( $G$ ”). (B) Complex

246 viscosity $\eta^{*}$ measured at a frequency of $1 \mathrm{~Hz}$ and $0.2 \%$ strain as a function of CMC

247 concentration in an aqueous suspension of MFC:CMC and also for MFC100 alone. For each

248 point the MFC concentrations are matched therefore the percentage of MFC in a pure solution 
252 A noticeable difference in complex viscosity was observed on comparing the MFC100 (ND) and MFC/CMC (ND) suspension (Figure 1B), this behaviour can be explained by the dilution effect of CMC on MFC producing the different ratios. Diluting the MFC network structure with CMC to make up the formulation (as per Table 1), results in less microfibril entanglement in the network structure and is also seen in the microscopy images presented in Figure 2A, resulting in a lower complex viscosity as compared with comparable concentrations of MFC100 (ND) (Figure 1B). A slightly lower complex viscosity was observed when comparing MFC/CMC (ND) and MFC/CMC (D) formulations, but this reduction was minimal in the case of CMC50 (Figure 1B). However, the CMC50 suspension showed weaker gel-like behaviour. The slight frequency dependence of the moduli and the relatively large value of $\tan \delta\left(\mathrm{G}^{\prime \prime} / \mathrm{G}^{\prime}\right.$ $>0.1$ ) defines so-called weak gel behaviour (Ikeda and Nishinari, 2001) as evident in Figure 1A. Tan $\delta$ values are also presented later in Figure 4 . When the negatively charged CMC was added at higher levels, the CMC adsorption to MFC increased significantly. Similar behaviour was reported with bacterial cellulose/CMC systems where changes in zeta-potential were shown (Veen et al., 2014). The increase in the charge for all ratios of CMC leads to better redispersibility of the MFC/CMC formulations in water with higher complex viscosity values. Lower values of G' where Tan $\delta>0.1$ for CMC50 suspensions can be explained by a dilution effect. As the dense network of microfibrils plays an important role in maintaining viscoelastic gel-like behaviour, when MFC is diluted with $50 \%$ CMC the MFC is at $1 \%$, which without additives shows an order of magnitude decrease in G' and G' (Figure 1B), and the MFC forms a weaker entangled network structure. 


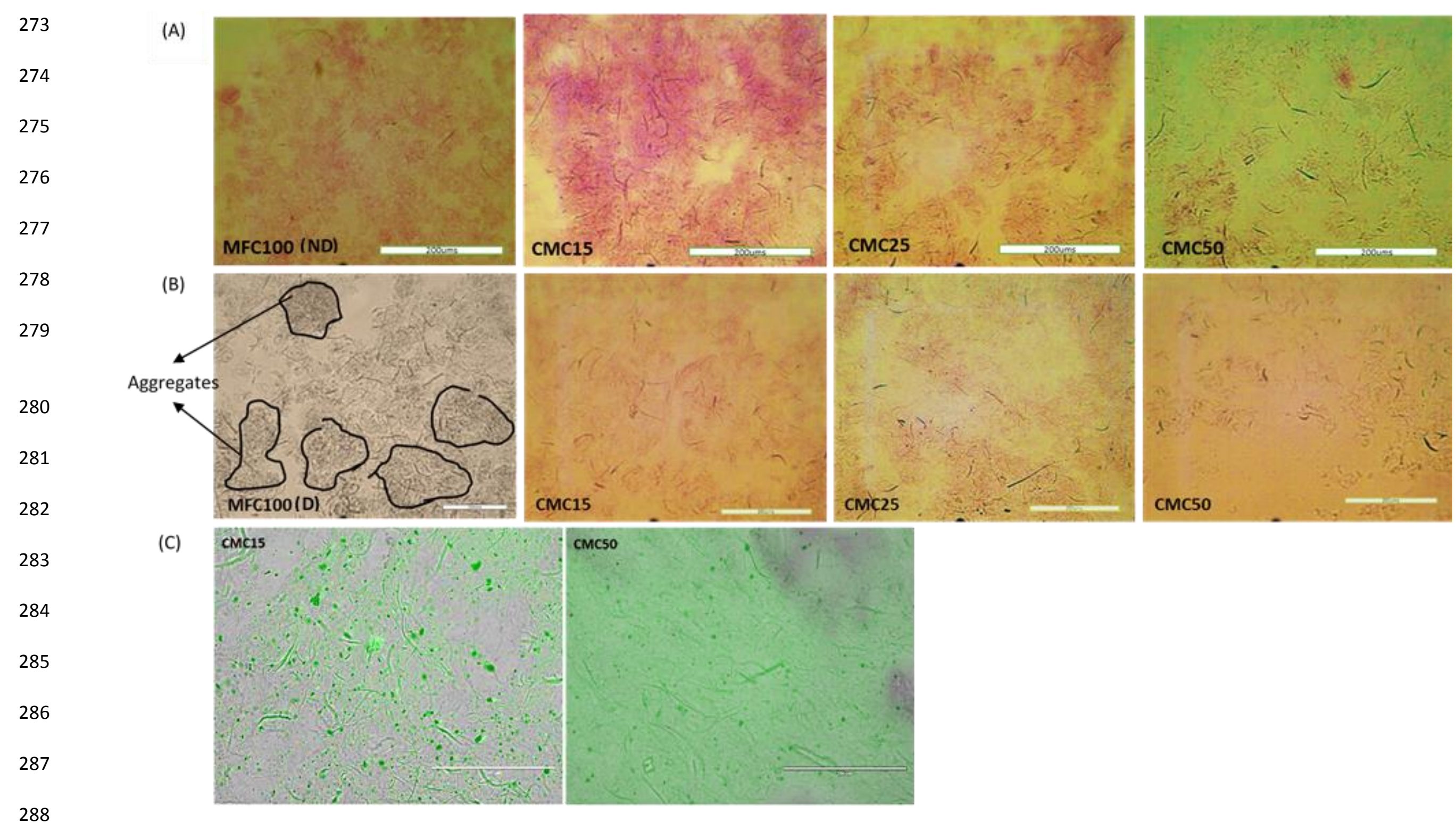


Figure 2 Light microscopy images of $2 \%$ w/w aqueous suspensions of $(A)$ never dried, (B)

290

291

292

293

294

295

296

297

298

299

300

301

302

303

304

305

306

307

308

309

310

311

312

313

dried and redispersed suspensions of MFC100 and MFC/CMC at CMC levels of 15, 25 and 50\%. (C) Fluorescence microscopy images of $2 \% \mathrm{w} / \mathrm{w}$ redispersed suspension of CMC15, and CMC50, scale bar 200um, where CMC is tagged with FITC (green fluorescence).

Light microscopy images of never-dried MFC with different levels of CMC indicated that the addition of CMC does not affect microfibrillar entangled network except at high levels (Figure 2A). A lower level of entanglement was observed in the case of CMC50. This can be explained by dilution effect of CMC on the MFC network structure as outlined for the case of complex viscosity earlier. Microscopy images of dried and redispersed MFC/CMC (i.e., CMC15, CMC25 \& CMC50) indicate that the addition of CMC reduced the microfibrillar aggregate or fibre bundle formation as compared to MFC100 (D) (Figure 2B). Drying MFC without CMC resulted in a large amount of microfibrillar aggregates due to the formation of strong inter- and intramolecular hydrogen bonds during the drying process (Figure 2B). These were difficult to redisperse in water and reduced the values of viscoelastic parameters such as G', G' and complex viscosities due to poor network formation. From fluorescence microscopy images (Figure 2C) it cannot be said with certainty that fluorescently tagged-CMC interacted at a molecular level with the surface of MFC microfibrils. It is strongly implied however from the comparison in Figure 2C of CMC15 and CMC50 that as the amount of CMC increased, either the surface coverage of MFC by CMC increased or there was a general build-up of the labelled CMC in the solution surrounding the fibres.

\subsection{Temperature dependence of the viscoelastic moduli}

The temperature dependence of $G^{\prime}$ and $G$ " for $2 \% \mathrm{w} / \mathrm{w}$ aqueous suspensions of MFC/CMC mixtures is shown in Figure 3. All the samples showed stable viscoelastic gel-like behaviour where the storage modulus was higher than the loss modulus throughout the temperature range $20^{\circ} \mathrm{C}-90^{\circ} \mathrm{C}$ at a heating rate of $1^{\circ} \mathrm{C} / \mathrm{min}$. It was observed that the $\mathrm{G}^{\prime}$ and $\mathrm{G}$ ” for all suspensions 
314 showed an initial slight decrease from $20^{\circ} \mathrm{C}$ to $40^{\circ} \mathrm{C}$, however above $40^{\circ} \mathrm{C}$ the suspensions

315 showed an increase in $\mathrm{G}^{\prime}$ and $\mathrm{G}^{\prime \prime}$ up to $90^{\circ} \mathrm{C}$. Similar behaviour was observed with cellulose nanofibers from poplar wood by Chen et al., 2013. The first slight decrease in modulus may be

317 due to thermal agitation/thermal motion of microfibrils, resulting in loosening of the fibrils within the network structure. However, the swelling of microfibrils with an increase in temperature, while interacting with $\mathrm{CMC}$ in the matrix, may strengthen the gel-like structure, resulting in an increased $\mathrm{G}^{\prime}$ and $\mathrm{G}$ " of suspensions above $40^{\circ} \mathrm{C}$. As the amount of $\mathrm{CMC}$ increased in the formulation, $\mathrm{G}^{\prime}$ and $\mathrm{G}$ ' increase to a greater extent above $40^{\circ} \mathrm{C}$ suggesting synergistic interactions between MFC/CMC. It is well known that polymeric solutions such as HPMC (hydroxyl propyl methyl cellulose), exhibit an increased thermal motion upon heating, leading to a weaker network and sometimes a decrease in viscosity, however the viscosity of these systems tends to increase above the gelation temperature depending on concentration (Silva et al., 2008). The fact that the MFC/CMC suspensions do not lose structure upon heating, even when the MFC proportion is lowered, indicates an interaction beyond the surface stabilisation of the microfibrils by $\mathrm{CMC}$, although it is not yet clear which mechanisms are involved.

331

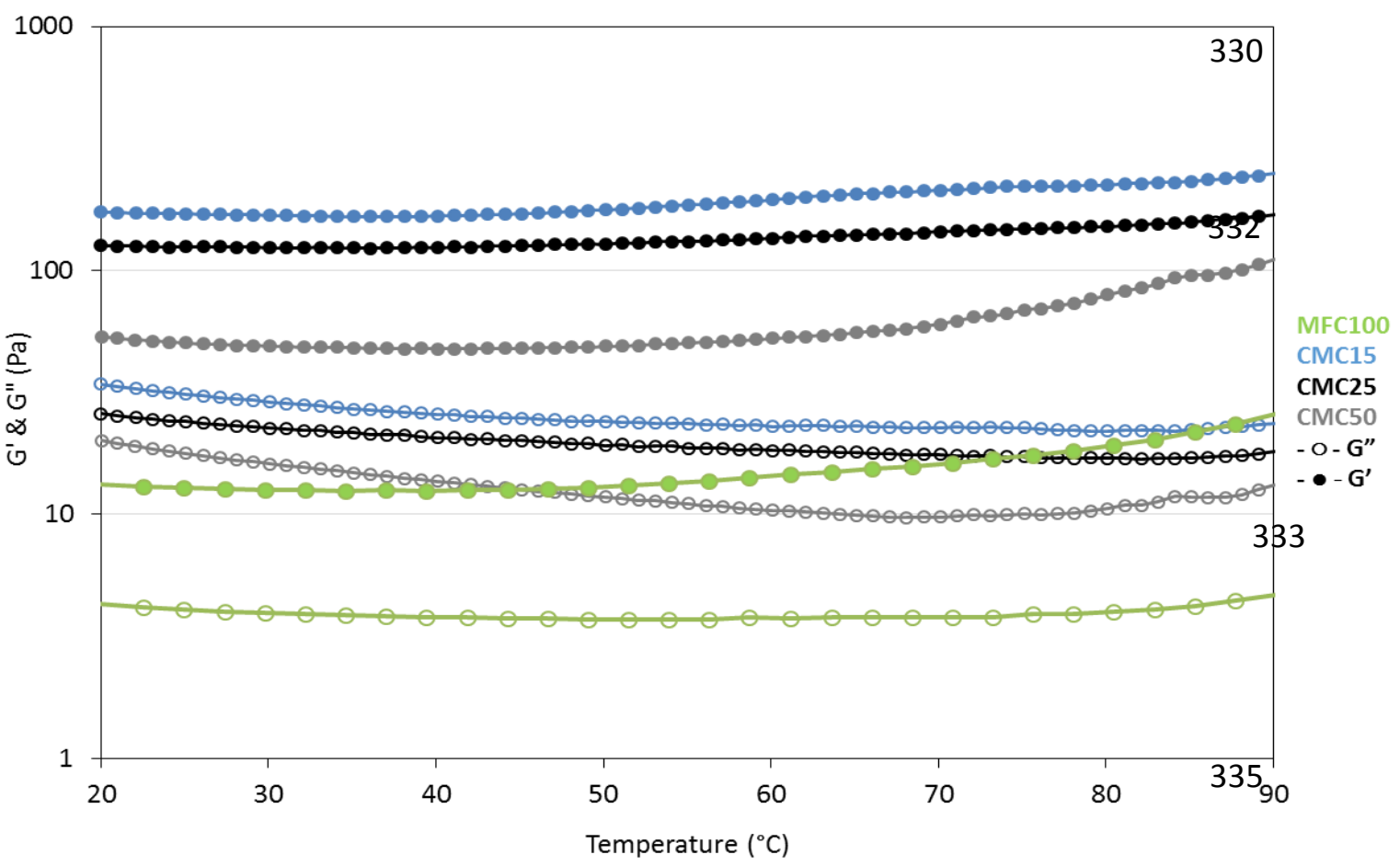



moduli of $2 \% \mathrm{w} / \mathrm{w}$ aqueous suspensions of MFC100 (D) and MFC/CMC (D) acquired at $1 \mathrm{~Hz}$ frequency and $1 \%$ strain. Solid symbols represent storage modulus (G') and open symbols represent loss modulus ( $G$ ").

\subsection{Relaxation time $\left(T_{2}\right)$ of $M F C / C M C$ suspensions}

Figure 4 shows the spin-spin relaxation time $\left(\mathrm{T}_{2}\right)$ as a function of the amount of CMC present in the MFC/CMC formulations. At higher levels of CMC in the formulation, the $\mathrm{T}_{2}(\mathrm{~ms})$ value and the Tan $\delta$ of the suspension increased, the latter implying that the suspension was behaving in a more viscous or liquid-like fashion. Lower $\mathrm{T}_{2}$ values for the redispersed MFC100 $(\mathrm{CMC}=0)$ suspensions are most likely due to the rigid network structure formed by strong intraor intermolecular $\mathrm{H}$-bond within the microfibrils and a consequently reduced $\mathrm{T}_{2}$ value for the polymeric component. It appears to be the presence of these rigid structures in case the of MFC100 (D) suspensions which dominate the $\mathrm{T}_{2}$ values at all concentrations. In this case, the overall $T_{2}$ value of the suspensions are driven by the $T_{2}$ value of the polymer " $1 / T_{2 p}$ " (see equation 1) assuming the water is behaving as bulk water and has not been perturbed in any way. The fraction of water which is proposed to be perturbed in such systems is normally low ( 2\%, McConnell \& Pope 2001).

$$
1 / \mathrm{T}_{2}=\mathrm{a}^{*}\left(1 / \mathrm{T}_{2 \mathrm{P}}\right)+(1-\mathrm{a})^{*}\left(1 / \mathrm{T}_{2 \mathrm{~W}}\right)
$$

Equation (1)

Equations of the form of equation 1 describe the effect of protons exchanging between a polymer site with the polymer present at a weight fraction $a$ and having a $\mathrm{T}_{2}$ value of $\mathrm{T}_{2 \mathrm{P}}$ and water at a weight fraction (1-a) having a $\mathrm{T}_{2}$ value of $\mathrm{T}_{2 \mathrm{w}}$ under conditions of a CPMG Tau value which allows exchange to be rapid. To examine the effect of drying on the overall apparent water mobility in the microfibrillar network in the presence and absence of CMC, the $\mathrm{T}_{2}$ values and shear viscosities as a function of concentration were plotted for aqueous 


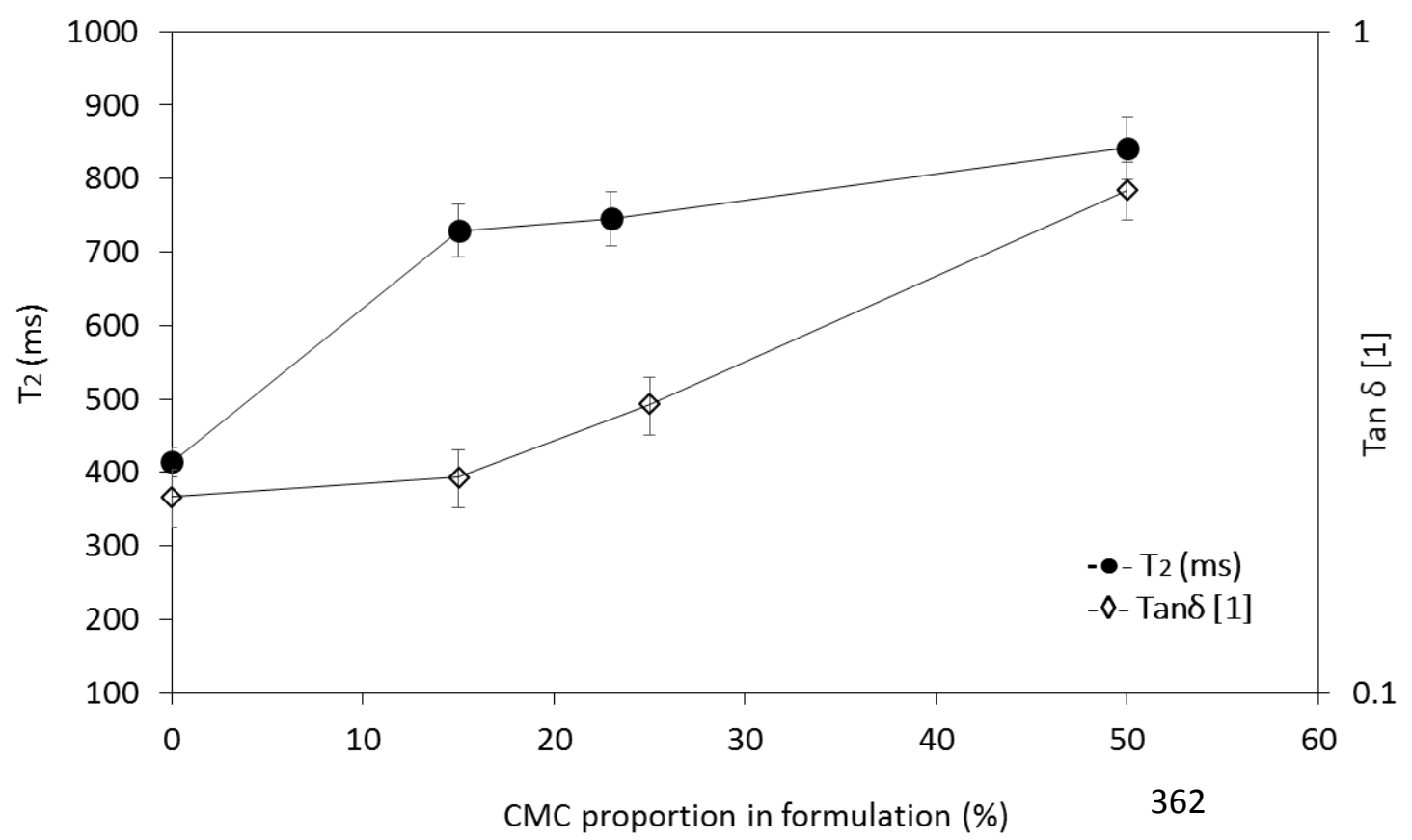

363 Figure 4 Change in $T_{2}(\mathrm{~ms})$ and Tan $\delta$ (measured at a frequency of $1 \mathrm{~Hz}$ and a strain of $0.2 \%$ ) plotted against increasing proportion of CMC in the suspension at $20^{\circ} \mathrm{C}$.

365 As suggested earlier, the drying of MFC without CMC results in the formation of strong intermolecular H-bonds between the microfibrils resulting in rigid fibre bundles or aggregates of MFC, which limits the polymer mobility within the microfibril network resulting in lower redispersibility. Effectively this reduces the $T_{2 p}$ value of the polymer and consequently increases the $1 / \mathrm{T}_{2 \mathrm{p}}$ value reducing the overall measured $\mathrm{T}_{2}$ as can be seen in figure $5 \mathrm{~B}$. As the concentration increases, this effect becomes more pronounced however now it is mediated by increases in the value of $a$. The net result is a further decrease in the value of $\mathrm{T}_{2}$. If the polymer is not dried then the bonding between the fibrillar complex is not as strong and the $\mathrm{T}_{2}$ values are higher by similar arguments to the above. Figure 5A shows that the addition of CMC to MFC i.e. CMC15 (D) significantly increases the shear viscosity of the redispersed suspension compared to MFC100 (D). Similarly, the overall $\mathrm{T}_{2}$ values of the redispersed CMC15 (D) were 
$377 \quad \mathrm{~T}_{2}$ values of all.

378

379

(A)

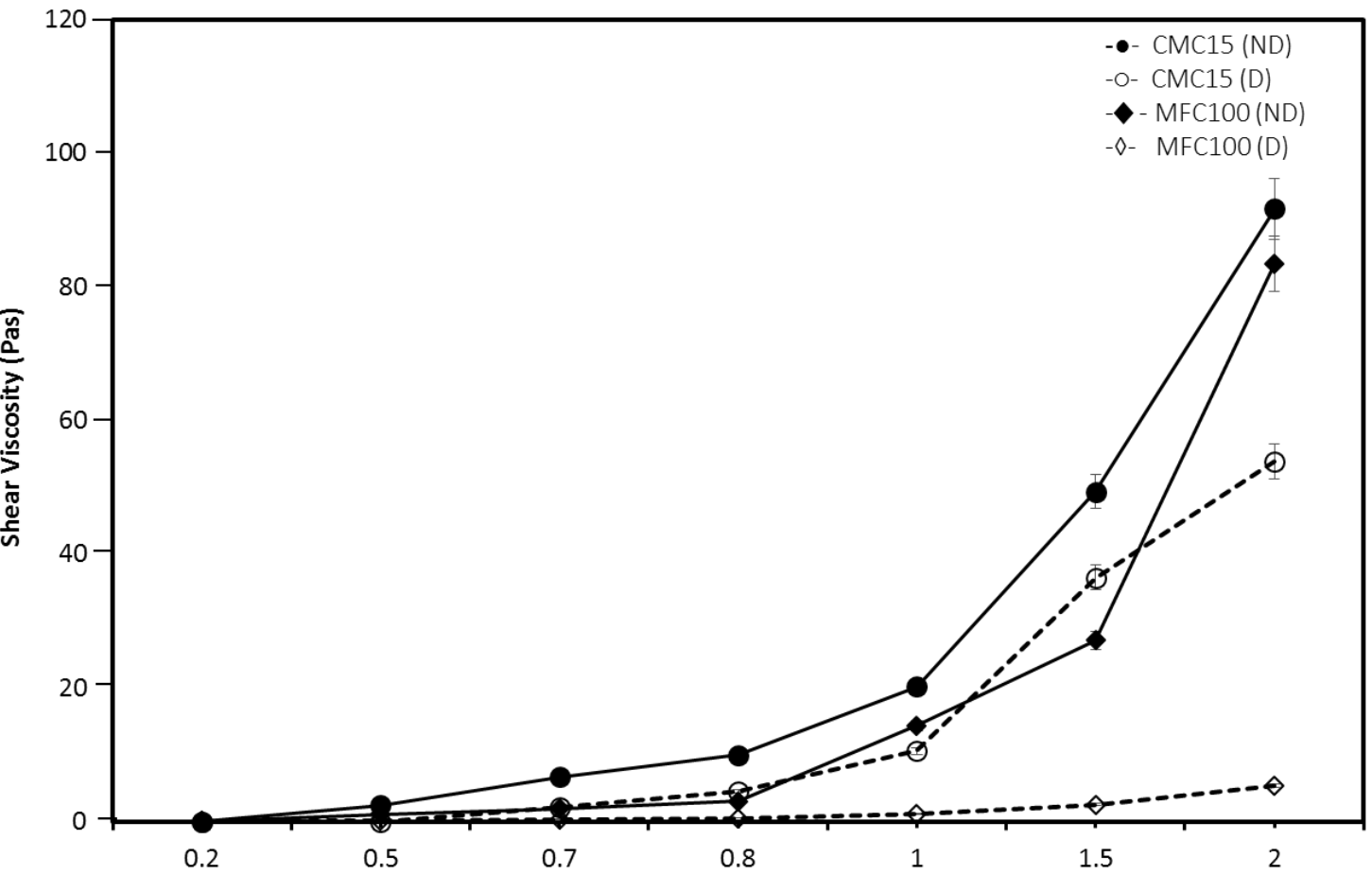

387

388

389

390

391

392

393

394

395

396

397

398

(B)

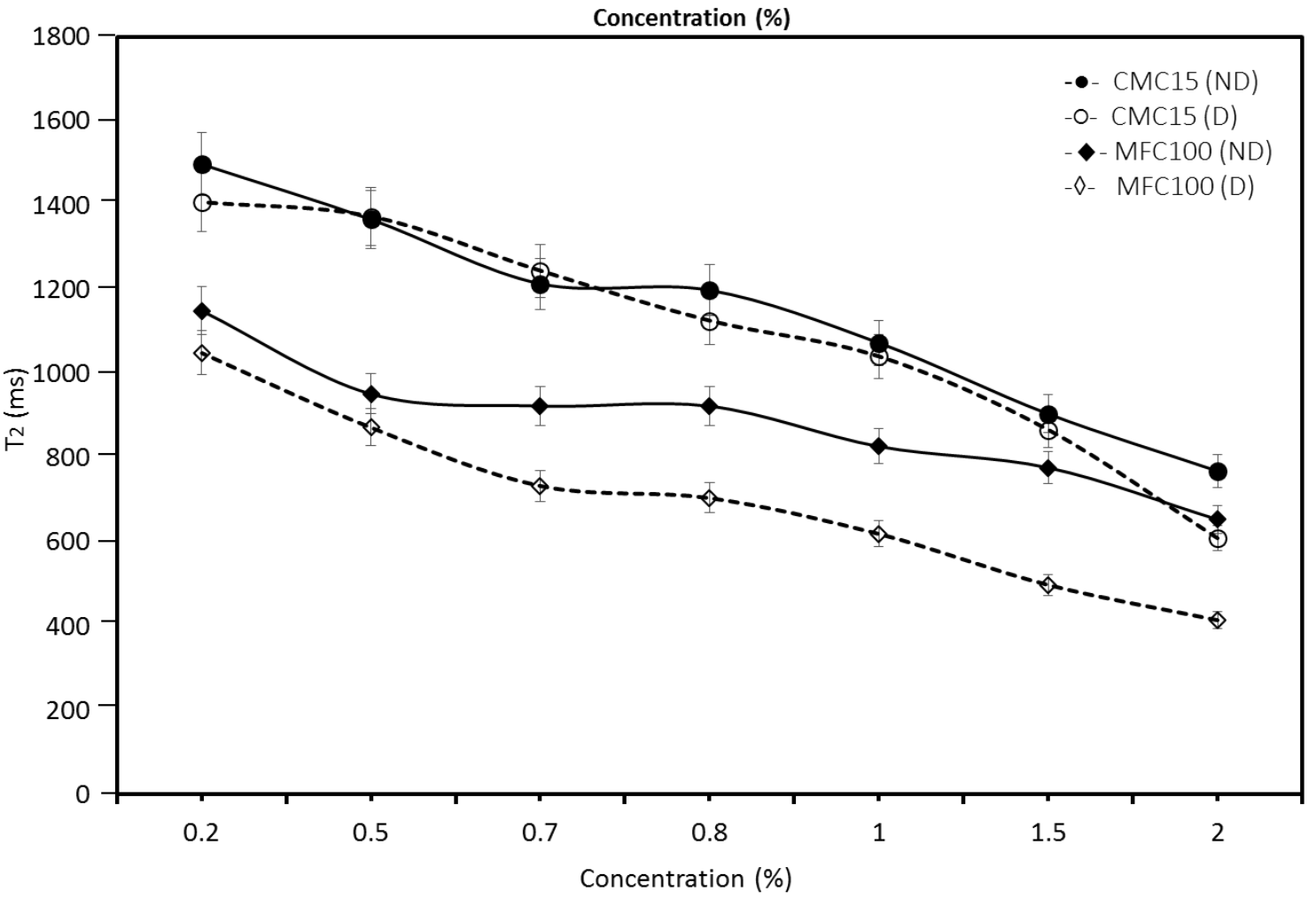

399

Figure 5 (A) Shear viscosity (at 50s ${ }^{-1}$ shear rate); and (B) Spin-spin relaxation time $T_{2}(\mathrm{~ms})$ as 400 a function of concentration at $20^{\circ} \mathrm{C}$ for $\left(-\nabla_{-}\right) \mathrm{MFC100}$ (ND) solid diamonds, (D) unfilled 401 diamonds; (-०-) CMC15 (ND) solid circles, (D) unfilled circles. 
The addition of CMC appears to prevent the formation of strong hydrogen bonds between MFC

403

404

405

406

407

408

409

410

411

412

413

414

415

416

417

418

419

420

421

422

423

424

425

fibres, resulting in improved redispersibility of the $\mathrm{CMC} 15$. The reduced amount of aggregates and fibres bundles in the redispersed suspension increases polymer mobility and hence increases the polymer $\mathrm{T}_{2}$ value in the $\mathrm{CMC} 15$. There may also be a direct effect of the CMC on the polymer via an altered ionic environment. The result of these changes is that the interactions between fibrils are weaker and the overall measured $\mathrm{T}_{2}$ increases. If this interpretation is correct then whilst the $\mathrm{NMR} \mathrm{T}_{2}$ value is sometimes loosely referred to as the water signal it is in fact actually only the apparent overall water mobility. Equation 1 gives a more accurate description of mobility in the system. In addition, because the bonds are now weakened by $\mathrm{CMC}$, the difference in $\mathrm{T}_{2}$ values between the dried and non-dried CMC containing materials is reduced as can be seen in Figure 5B. Drying the MFC100 systems results in tighter bonding which impairs redispersibility and results in substantial differences between dried and non-dried MFC100.

\section{Conclusions}

The influence of CMC on the rheological properties of MFC suspension is consistent with an exchange based NMR interpretation of spin-spin relaxation times $\left(\mathrm{T}_{2}\right)$ for polymer and water. Rheological measurements show that addition of CMC to MFC increases complex viscosity and shear viscosity of the suspension compared to dried MFC without additives. Fluorescence microscopy showed that the CMC tends to interact homogenously with MFC possibly on the surface of the microfibrils present in the network. This prevents the formation of H-bonds between the MFC's microfibrils, hence making dried MFC/CMC easier to redisperse in water. The lower $\mathrm{T}_{2}$-values of the single component $\mathrm{MFC100}$ suspensions result from the rigid structures formed upon drying and the lower polymer mobility. The addition of CMC to the MFC suspensions improved redispersibility of MFC after drying and produces stable and 
427 the matrix.

\section{Acknowledgement}

429 This work was supported by the Oslofjordfond, Norway grant scholarship (2012 - 2015).

\section{$430 \quad$ References}

431 Agoda-Tandjawa G., Durand S., Berot S., Blassel C., Gaillard C., Garnier C., Doublier L.J. 432 (2010) Rheological characterization of microfibrillated cellulose suspensions after freezing. 433 Carbohydrate Polymers 80, 677-686.

434 Agoda-Tandjawa G., Durand S., Gaillard C., Garnier C, Doublier J.L., (2012) Rheological 435 behaviour and microstructure of microfibrillated cellulose suspensions/low-methoxyl pectin 436 mixed systems. Effect of calcium ions. Carbohydrate Polymers. 87-2, 1045-1057.

437 Andresen M., Stenstad P., Møretrø T., Langsrud S., Syverud K., Johansson L.S., Stenius P. 438 (2007). Nonleaching Antimicrobial Films Prepared from Surface-Modified Microfibrillated 439 Cellulose. Biomacromolecules. 8-7, 2149-2155

440 Belder De. N.A, Granath K. (1973) Preparation and properties of fluorescein-labelled dextrans. 441 Carbohydrates Research. 30, 375-378.

442 Bruce D.M., Hobson R.N., Farrent J.W., Hepworth D.G. (2005). High-performance composites 443 from low-cost plant primary cell walls. Composites Part A: Applied science and 444 Manufacturing. 36-11, 1486-1493.

445 Burger C., Hsiao B.S., Chu B. (2006). Nanofibrous Materials and Their Applications. Annu 446 Rev Mater Res. 36, 333-368.

447 Chen P., Yu H., Liu Y., Chen W., Wang X., Ouyang M. (2013) Concentration effects on the 448 isolation and dynamic rheological behaviour of cellulose nanofibers via ultrasonic processing. 449 Cellulose. 20,149-157.

450 Cordoba A., Camacho M.D.M., Navarrete N.M. (2010). Rheological behaviour of an insoluble 451 lemon fibre as affected by stirring, temperature, time and storage. Food and Bioprocess 452 Technology. 5-3, 1083-1092. 
453 Fernandes D.J.M.B., Gil M.H., Castro J.A.A.M. (2004). Hornification-its origin and 454 interpretation in wood pulps. Wood Science and Technology. 37, 489-494.

455 Frone, A. N., Panaitescu, D. M., \& Donescu, D. (2011). Some aspects concerning the isolation 456 of cellulose micro- and nano-fibers. U.P.B. Science Bulletin, Series B. 73-2, 133-152.

457 Henriksson M., Henriksson, G., Berglund, L. A., \& Lindstrom, T. (2007) An environmentally 458 friendly method for enzyme-assisted preparation of microfibrillated cellulose (MFC) 459 nanofibers. European Polymer Journal, 43, 3434-3441.

460 Herrick F.W., Casebier R.L., Hamilton J.K,. Sandberg K.R. (1983). Microfibrillated cellulose: 461 morphology and accessibility. J Appl Polym Sci: Appl Polym Symp. 37-9,797-813.

462 Ikeda S., Nishinari K. (2001). “Weak Gel”-Type Rheological Properties of Aqueous 463 Dispersions of Non-aggregated K-Carrageenan Helices. Journal of Agriculture Food 464 Chemistry. 49, 4436-4441.

465 Kato K.L., Cameron R.E., (1999). A review of the relationship between thermally-accelerated 466 ageing of paper and hornification. Cellulose. 6, 23-40.

467 Khopade A.J., Jain N.K. (1990). A Stable Multiple Emulsion System Bearing Isoniazid: 468 Preparation and Characterization. Drug Dev Ind Pharm. 24-3, 289-293.

469 Kirk R.E., Othmer D.F. Cellulose, Encylopedia of chemical technology (2nd ed.) Wiley, New 470 York. 4

471 Kocherbitov V., Ulvenlund S., Kober M., Jarring K., Arnebrant T. (2008) Hydration of 472 microcrystalline cellulose and milled cellulose studied by sorption calorimetry. J. 473 Phys.Chem.B. 112. 3728-3734.

474 Lavoine N., Desloges I., Dufresne A., Bras J. (2012). Microfibrillated cellulose - Its barrier 475 properties and applications in cellulosic materials: A review. Carbohydrate Polymers. 90-2, $476735-764$.

477 López-Rubio A, Lagaron J.M., Ankerfors M., Lindström T., Nordqvist D., Mattozzi A., 478 Hedenqvist M.S. (2007). Enhanced film forming and film properties of amylopectin using 479 micro-fibrillated cellulose. Carbohydrate Polymers. 68-4,718-727.

480 Lowys M.P., Desbrières, J., Rinaudo, M. (2001) Rheological characterization of cellulosic 481 microfibril suspensions: Role of polymeric additives. Food Hydrocolloids. 15,25-32. 
Malainine M.E., Mahrouz M., Dufresne A. (2005). Thermoplastic nanocomposites based on cellulose microfibrils from Opuntia ficus-indica parenchyma cell. Composites Science Technology. 65-10, 1520-1526.

McConville P, Pope J.M. (2001). 1H NMR T2 relaxation in contact lens hydrogels as a probe of water mobility. Polymer. 42(8), 3559-3568.

Meiboom S. and Gill, D (1958) Modified spin-echo method for measuring nuclear relaxation times. Rev. Sci. Instrum. 29, 688-691.

Missoum K., Bras J., Belgacem N.M., (2012). Water redispersible dried nanofibrillated cellulose by adding sodium chloride. Biomacromolecules. 13, 4118-4125.

Nakagaito A.N, Yano H. (2004). The effect of morphological changes from pulp fiber towards nano-scale fibrillated cellulose on the mechanical properties of high-strength plant fiber based composites. Appl Phys A: Mater Sci Process. 78-4, 547-552.

Nishiyama Y., (2009) Structure and properties of the cellulose microfibril. J Wood Sci. 55,241249.

Norton I.T., Foster, T.J., Brown, R. (1998). The science and technology of fluid gels. Gums and stabilisers for the food industry. Ed. William, P.A., Phillips, G.O. Edition 9; 259-268.

Ono H., Inamoto M., Okajima K., (1997) Spin-lattice relaxation behaviour of water in cellulose materials in relation to the tablet forming ability of microcrystalline cellulose particles. Cellulose. 4, 57-73.

Ougiya H., Watanabe K., Morinaga Y., Yoshinaga F. (1997). Emulsion-stabilizing Effect of Bacterial Cellulose. Biosci Biotechnol Biochem. 61-9, 1541-1545.

Oza K.P., Frank S.G.J.J. (1986). Microcrystalline Cellulose Stabilized emulsions. Dispersion Sci Technol. 7-5, 543-561.

Pääkkö M., Ankerfors M., Kosonen H., Nykänen A., Ahola S., Österberg M., Ruokolainen J., Laine J., Larsson P.T., Ikkala O., and Lindström T. (2007). Enzymatic Hydrolysis Combined with Mechanical Shearing and High-Pressure Homogenization for Nano-scale Cellulose Fibrils and Strong Gels. Biomacromolecules. 8, 1934-1941. 
Quievy N., Jacquet N., Sclavons M., Deroanne C., Paquot M., Devaux J. (2010) Influence of 510 homogenization and drying on the thermal stability of microfibrillated cellulose. Polymer degradation and stability. 95,306-314.

512 Rachocki A., Markiewicz E., Goc-Tritt J. (2005) Dielectric relaxation in cellulose and its 513 derivatives. Acta Physica Polonica A. 108, 137-145.

514 Ribitsch V., Stana-Kleinschek K., Kreze T., and Strnad S. (2001). The significance of surface 515 charge and structure on the accessibility of cellulose fibres. Macromolecular Materials and 516 Engineering. 286, 648-654.

517 Silva S.M.C., Pinto, F.V., Antunes, F.E., Miguel, M.G., Sousa, J.J.S., Pais, A.A.C.C. (2008). 518 Aggregation and gelation in hydropropylmethyl cellulose aqueous solutions. Journal of Colloid 519 and Interface Science. 327, 333-340.

520 Smook G.A. (1990) Hornification (keyword). In: Handbook of pulp \& paper terminology. 521 Angus Wilde, Vancouver, p 135.

522 Syverud K., Stenius P. (2009). Strength and barrier properties of MFC films. Cellulose 16, 7552385.

524 Tatsumi D., Ishioka S., \& Matsumoto T. (2002). Effect of fibre concentration and axial ratio 525 on the rheological properties of cellulose fibre suspensions. Journal of the Society of Rheology 526 Japan. 30-1, 27-32.

527 Tatsumi, D. (2007). Rheology of cellulose fibre disperse systems and cellulose solutions. 528 Nihon Reoroji Gakkaishi. 35-5, 251-256.

529 Turbak A.F., Snyder F.W., Sandberg K.R. (1983). Microfibrillated cellulose, a new cellulose 530 product: properties, uses, and commercial potential. J Appl Polym Sci: Appl Polym Symp. 37, 531815.

532 Vackier C.M., Hills B.P., Rutledge D.N. (1999) An NMR Relaxation study of the state of water 533 in gelatin gels. Journal of Magnetic Resonance. 138, 36-42.

534 Veen S.J., Kuijk, A., Versluis, P., Husken, H., Krassimir, P.V. (2014). Phase transitions in 535 cellulose microfibril dispersions by high-energy mechanical de-agglomeration. Langmuir. 30, $536 \quad 13362-13368$. 